ARTICLE

Received 16 Jun 2015 | Accepted 23 Mar 2016 | Published 26 Apr 2016

DOI: $10.1038 /$ ncomms11421

OPEN

\title{
Strong nonlinear terahertz response induced by Dirac surface states in $\mathrm{Bi}_{2} \mathrm{Se}_{3}$ topological insulator
}

Flavio Giorgianni ${ }^{1}$, Enrica Chiadroni ${ }^{2}$, Andrea Rovere ${ }^{1}$, Mariangela Cestelli-Guidi ${ }^{2}$, Andrea Perucchi $^{3}$, Marco Bellaveglia ${ }^{2}$, Michele Castellano², Domenico Di Giovenale², Giampiero Di Pirro², Massimo Ferrario², Riccardo Pompili ${ }^{2}$, Cristina Vaccarezza ${ }^{2}$, Fabio Villa ${ }^{2}$, Alessandro Cianchi ${ }^{4}$, Andrea Mostacci ${ }^{5}$, Massimo Petrarca ${ }^{5}$, Matthew Brahlek ${ }^{6}$, Nikesh Koirala ${ }^{6}$, Seongshik Oh ${ }^{6}$ \& Stefano Lupi ${ }^{1}$

Electrons with a linear energy/momentum dispersion are called massless Dirac electrons and represent the low-energy excitations in exotic materials such as graphene and topological insulators. Dirac electrons are characterized by notable properties such as a high mobility, a tunable density and, in topological insulators, a protection against backscattering through the spin-momentum locking mechanism. All those properties make graphene and topological insulators appealing for plasmonics applications. However, Dirac electrons are expected to present also a strong nonlinear optical behaviour. This should mirror in phenomena such as electromagnetic-induced transparency and harmonic generation. Here we demonstrate that in $\mathrm{Bi}_{2} \mathrm{Se}_{3}$ topological insulator, an electromagnetic-induced transparency is achieved under the application of a strong terahertz electric field. This effect, concomitantly determined by harmonic generation and charge-mobility reduction, is exclusively related to the presence of Dirac electron at the surface of $\mathrm{Bi}_{2} \mathrm{Se}_{3}$, and opens the road towards tunable terahertz nonlinear optical devices based on topological insulator materials.

\footnotetext{
${ }^{1}$ INFN and Dipartimento di Fisica, Università di Roma 'La Sapienza', Piazzale A. Moro 2, I-00185 Roma, Italy. ${ }^{2}$ Laboratori Nazionali di Frascati-INFN, Via E. Fermi, 40, I-00044 Frascati, Italy. ${ }^{3}$ INSTM Udr Trieste-ST and Elettra-Sincrotrone Trieste S.C.p.A, Area Science Park, I-34012 Trieste, Italy. ${ }^{4}$ INFN and Dipartimento di Fisica, Università di Roma 'Tor Vergata', viale della Ricerca Scientifica 1, I-00133 Roma, Italy. ${ }^{5}$ INFN and Dipartimento S.B.A.I., Università di Roma 'La Sapienza', Piazzale A. Moro 2, I-00185 Roma, Italy. ${ }^{6}$ Department of Physics and Astronomy Rutgers, The State University of New Jersey, 136 Frelinghuysen Road, Piscataway, New Jersey 08854-8019, USA. Correspondence and requests for materials should be addressed to S.L. (email: stefano.lupi@roma1.infn.it).
} 
$\mathrm{N}$ onlinear optical phenomena have a crucial importance in modern physics giving rise to fundamental applications such as coherent control of excitations in condensed matter, and harmonic generation and frequency conversion in optically active materials ${ }^{1-3}$. In this case, the use of materials whose electromagnetic response can be fully controlled by an applied radiation field plays a fundamental role in ultrafast electromagnetic pulse generation and shaping ${ }^{4}$. These nonlinear optical properties, earlier discovered and studied in the visible and near-infrared range of the electromagnetic spectrum, have been successively investigated at infrared frequencies 5 , and their extension towards the terahertz $(\mathrm{THz})$ region $\left(1 \mathrm{THz}=33 \mathrm{~cm}^{-1}=300 \mu \mathrm{m}=4 \mathrm{meV}\right)$, a spectral range that has seen recently a tumultuous technological and scientific development ${ }^{6}$, is highly desirable. $\mathrm{THz}$ research has been oriented either on investigating novel radiation sources based on frequency conversion, optical rectification ${ }^{7-9}$ and relativistic electrons $s^{10-15}$, and in studying the properties of plasmon-based systems whose optical properties such as absorption, dispersion and scattering can be engineered at $\mathrm{THz}$ frequencies ${ }^{16-18}$. As a matter of fact, the discovery of natural materials showing exotic nonlinear $\mathrm{THz}$ properties could set a new agenda in photonic and plasmonic applications of $\mathrm{THz}$ radiation.

One of the first observations of a nonlinear $\mathrm{THz}$ effect has been achieved in doped semiconductors by means of a $\mathrm{THz}$ electric field in the hundred of $\mathrm{kV} \mathrm{cm}^{-1}$ range ${ }^{19,20}$. In GaAs, for instance, free-charge carriers introduced by doping or thermally excited in the conduction band can be accelerated by the $\mathrm{THz}$ electric field. When the momentum gain is sufficiently high, carriers are scattered from the bottom of the conduction band into satellite valleys. Here electrons show an increased effective mass with respect to low-lying states, leading to a reduction of carrier mobility and then to an enhanced $\mathrm{THz}$ transparency. A similar band-structure effect has been observed in $\mathrm{Si}$ and $\mathrm{Ge}$, giving rise to a comparable $\mathrm{THz}$-induced transparency ${ }^{21}$.

Recently, theoretical models have predicted a strong nonlinear $\mathrm{THz}$ response of two-dimensional (2D) metallic systems characterized by massless Dirac electrons. Their nonlinear response has been estimated to be higher than massive electron plasma in conventional metals ${ }^{22-24}$. This strong nonlinearity can be qualitatively understood in terms of a simple calculation ${ }^{23}$. Consider charge carriers having a Dirac dispersion $\epsilon(p)=\mathbf{V}_{\mathrm{F}} \cdot \mathbf{p}=V_{\mathrm{F}} \sqrt{p_{x}^{2}+p_{y}^{2}}$, where $\mathbf{p}$ is the linear momentum and $\mathbf{V}_{\mathrm{F}}$ is the Fermi velocity. Under an oscillating $\mathrm{THz}$ electric field $E(t)=E_{0}$ cosvt, electrons gain (for zero scattering) a momentum $\mathbf{p}(t)=-\frac{e E_{0}}{v} \sin v t$. The band velocity (for instance along the $x$ direction) can be calculated by the equation $V_{x}=\partial \epsilon / \partial p_{x}$ and, for vanishing $p_{y}, V_{x}(t)=-V_{\mathrm{F}} \operatorname{sgn}(\sin v t)$. Therefore, if $n_{\mathrm{D}}$ is the Dirac surface density and neglecting the statistical distribution of carriers, this corresponds to an intraband Dirac current $J_{\mathrm{D}}(t)=e n_{\mathrm{D}} V_{\mathrm{F}} \operatorname{sgn}(\sin v t)=$ $(4 / \pi) \mathrm{e} n_{\mathrm{D}} V_{\mathrm{F}}[\sin v t+1 / 3 \quad \sin 3 v t+1 / 5 \quad \sin 5 v t+\ldots .],$.$\quad which$ contains all odd harmonics $J_{m}(m=1,3,5, \ldots)$, with an amplitude decreasing as $1 / m$ (ref. 24). Although both the presence of scattering and the actual statistical distribution of electrons may modify the harmonic intensity ratio as theoretically calculated $^{25-27}$ and experimentally observed in graphene $e^{28}$, the previous result still remains valid, and it is exclusively due to the peculiar charge-carrier Dirac energy/momentum dispersion.

Graphene is the first material in which massless Dirac carriers have been predicted theoretically and soon experimentally observed $^{29,30}$. Here different experiments have further shown sizable nonlinear optical effects at $\mathrm{THz}$ frequency ${ }^{28,31-34}$. However, Dirac electrons have been recently discovered in many other materials such as three-dimensional (3D) Dirac semimetals $\mathrm{Cd}_{3} \mathrm{As}_{2}$ and $\mathrm{NaI}_{3}$ (refs 35,36), giving rise to intensive investigations of their intriguing properties. Perhaps, one of the most important classes of Dirac systems is represented by 3D topological insulators. These materials are quantum systems characterized by an insulating electronic gap in the bulk, whose opening is due to strong spin-orbit interaction, and gapless surface states at their interfaces ${ }^{37}$. Surface states in topological insulators are metallic, characterized by a Dirac dispersion, showing a chiral spin texture ${ }^{38,39}$ and protected from backscattering by the time-reversal symmetry. Since their discovery, topological insulators have attracted a growing interest due to their potential application in quantum computing $^{40,41}, \mathrm{THz}$ detectors ${ }^{42}$ and spintronic devices ${ }^{43}$. Linear $\mathrm{THz}$ spectroscopy has been applied to 3D topological insulators, in particular on thin films of $\mathrm{HgTe}$ and $\mathrm{Bi}_{2} \mathrm{Se}_{3}$, and their both Dirac single particle ${ }^{44-47}$ and collective (plasmon) ${ }^{48-50}$ excitations versus temperature and applied magnetic field have been observed. However, to our knowledge, $\mathrm{THz}$ nonlinear electrodynamics properties have been never investigated on 3D topological insulators so far. In this paper, we fill this gap, reporting on the experimental observation of a strong nonlinear $\mathrm{THz}$ absorption in $\mathrm{Bi}_{2} \mathrm{Se}_{3}$ topological insulator thin films. Their electromagnetic response has been studied over seven decades of $\mathrm{THz}$ electric field amplitude (from $0.1 \mathrm{~V} \mathrm{~cm}^{-1}$ to $1.5 \mathrm{MV} \mathrm{cm}^{-1}$ ) by combining linear spectroscopy through conventional $\mathrm{THz}$ radiation with data achieved at the high-intensity SPARC_LAB linac-based $\mathrm{THz}$ source in Italy. This source delivers broadband highly intense $\mathrm{THz}$ pulses with femtosecond shaping ${ }^{12,14}$. In $\mathrm{Bi}_{2} \mathrm{Se}_{3}$ topological insulator, we observe an induced electromagnetic transparency that increases and eventually saturates at high $\mathrm{THz}$ electric fields. This nonlinear $\mathrm{THz}$ behaviour is associated with the presence of Dirac electrons onto the surfaces. Indeed, in $\left(\mathrm{Bi}_{0.9} \mathrm{In}_{0.1}\right)_{2} \mathrm{Se}_{3}$, a material having the same crystal structure of $\mathrm{Bi}_{2} \mathrm{Se}_{3}$ and showing instead a trivial topology characterized by a gas of massive (Schrödinger) electrons at the surface $^{51}$, we do not observe any nonlinear effect in the same electric field interval.

\section{Results}

Grown and characterization of $\mathbf{B i}_{2} \mathrm{Se}_{3}$ thin films. Two thin films of $\mathrm{Bi}_{2} \mathrm{Se}_{3}$ were grown by molecular beam epitaxy on a 0.5-mm-thick sapphire $\left(\mathrm{Al}_{2} \mathrm{O}_{3}\right)$ substrate. One film had a thickness $t=120$ quintuple layers (QLs), where $1 \mathrm{QL} \simeq 1 \mathrm{~nm}$, the other one $t=60 \mathrm{QL}$ (refs 52,53). An additional film doped by In, $\left(\mathrm{Bi}_{0.9} \mathrm{In}_{0.1}\right)_{2} \mathrm{Se}_{3}$, with $t=60 \mathrm{QL}$, which shows a trivial topology and a massive (Schrödinger) electron gas with a similar surface density of $\mathrm{Bi}_{2} \mathrm{Se}_{3}$ (ref. 51), was grown on the same substrate for a sake of comparison. Each film was preliminarily characterized by transport and Hall measurements ${ }^{52,53}$. Their linear THz response was further investigated by Fourier transform spectroscopy 48,49 (Supplementary Note 1).

Nonlinear THz measurements. In Fig. 1, the experimental set-up is reported. Highly intense sub-picosecond $\mathrm{THz}$ pulses are produced at SPARC_LAB as coherent transition radiation (CTR) emitted by ultra-short $(\simeq 120 \mathrm{fs})$ high-brightness electron bunches ${ }^{11,14}$. THz radiation is reflected at $90^{\circ}$ with respect to the electron beam direction and transmitted through a $z$-cut quartz window to an off-axis parabolic mirror. This mirror produces collimated radiation that is further reflected by a flat mirror at $45^{\circ}$ and finally focalized on the film surface by a third off-axis parabolic mirror. A silicon beam splitter mounted before the films uses a portion of the beam to implement a differential detection system, allowing to remove the shot-by-shot fluctuation effects of the source. Finally, a pair of parallel wiregrid polarizers was used 


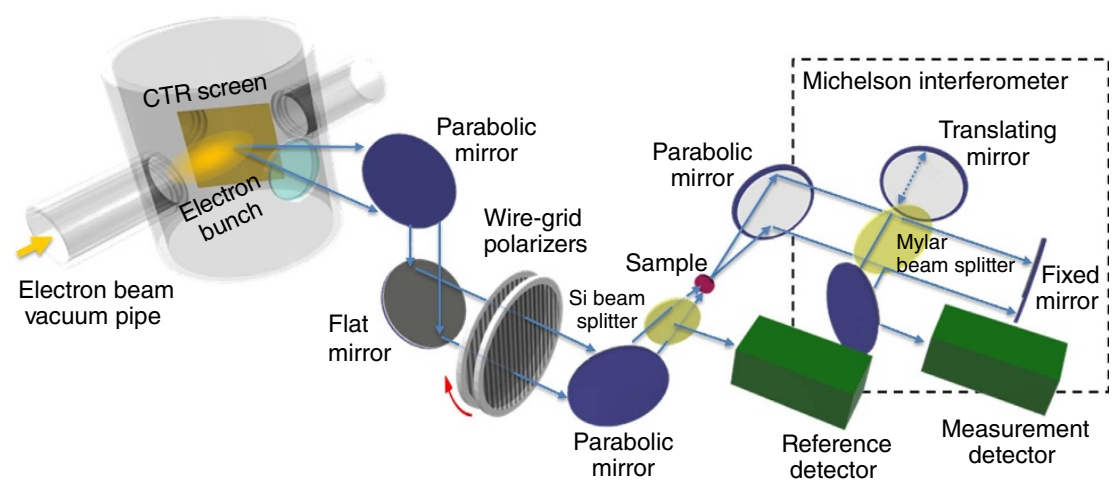

Figure 1 | Scheme of experimental set-up at SPARC terahertz source. Ultra-short electron bunches, from the high-brightness photoinjector, interacting with a metallic screen produce highly intense sub-picosecond coherent transition radiation $\mathrm{THz}$ pulses. $\mathrm{THz}$ radiation (blue arrows), emitted at $90^{\circ}$ with respect the electron propagation direction, is transmitted by a z-cut quartz window, and collected and collimated by means of an axis-off parabolic mirror. A further flat mirror was used to reflect the $\mathrm{THz}$ radiation up to the optical table, where a second off-axis parabolic mirror focalized the THz pulses on film samples. A pair of parallel wiregrid polarizers (QMC Inc.) have been used to tune the amplitude of the $\mathrm{THz}$ electric field over four decades: from $1 \mathrm{kV} \mathrm{cm}{ }^{-1}$ to $1.5 \mathrm{MV} \mathrm{cm}^{-1}$. A further, twin, off-axis parabolic mirror is finally used for illuminating a Michelson interferometer equipped with a GENTEC-EO pyroelectric detector that has been used for measuring the spectrally resolved transmittance. A further GENTEC-EO pyroelectric detector (reference detector) was mounted before the films to implement a differential detection to remove the shot-by-shot fluctuation effects of the SPARC THz source. Integrated transmittances were measured substituting the Michelson interferometer with another GENTEC-EO pyroelectric detector that is mounted just behind the films.

to tune the amplitude of $\mathrm{THz}$ electric field over four decades: from $1 \mathrm{kV} \mathrm{cm}^{-1}$ to $1.5 \mathrm{MV} \mathrm{cm}^{-1}$. We performed both integrated transmittance measurements (through a pyroelectric detector mounted behind the samples that collects the $\mathrm{THz}$ intensity transmitted by the film (substrate)) and spectrally resolved ones using a step-scan Michelson interferometer (Fig. 1). Integrated and spectrally resolved transmittance at lower electric fields (from 0.1 to $1 \mathrm{~V} \mathrm{~cm}^{-1}$ ) was measured by Fourier transform spectroscopy with a conventional mercury lamp. As film transmittances have been normalized to the transmittances of the bare substrate, we have verified that the sapphire substrate response is independent of the amplitude of the $\mathrm{THz}$ field. Therefore, the nonlinear $\mathrm{THz}$ effects here observed are exclusively due to the $\mathrm{Bi}_{2} \mathrm{Se}_{3}$ topological insulator films.

Figure 2a,b shows the integrated transmittance at $300 \mathrm{~K}$, normalized to that of substrate versus the $\mathrm{THz}$ electric field amplitude $E_{0}$ for the $60-\mathrm{QL}$ and $120-\mathrm{QL} \mathrm{Bi}_{2} \mathrm{Se}_{3}$ films. From 0.1 to $50 \mathrm{kV} \mathrm{cm}^{-1}$ the integrated transmittance (on the order of $60 \%$ for $60 \mathrm{QL}$ and $50 \%$ for $120 \mathrm{QL}$ ) does not change appreciably. Therefore, this electric field range corresponds to the linear region in which the optical properties of $\mathrm{Bi}_{2} \mathrm{Se}_{3}$ are practically independent of the $\mathrm{THz}$ field. For fields above this range, the integrated transmittance follows a monotonous increasing behaviour, indicating an enhancement of transparency. For fields above $1 \mathrm{MV} \mathrm{cm}^{-1}$, instead it begins to saturate to a value of $70 \%$ (63\%) for 60-QL (120-QL) film, corresponding to an enhanced transparency of $\sim 20 \%$ with respect to the value in the linear region.

In the insets of Fig. 2a,b, we show the frequency-resolved transmittance for both the samples at three different electric field amplitudes: $0.1 \mathrm{~V} \mathrm{~cm}^{-1}$ (as measured by a Michelson interferometer coupled with a mercury source), 0.5 and $1 \mathrm{MV} \mathrm{cm}^{-1}$ (these last values by the $\mathrm{THz}$ source at SPARC_LAB). At the lowest field, both the films show a transmittance that slightly decreases for frequency $v \rightarrow 0$. This behaviour is the signature of a free electron (Drude) absorption, which is associated mainly with Dirac surface states 46,47 , although a contribution from a $2 \mathrm{D}$ massive electron gas due to band-bending effects, cannot be ruled out, in particular at room- $T^{54}$. Moreover, in the high-frequency part of the transmittance spectrum $(\sim 1.8 \mathrm{THz})$, one observes a minimum that is related to the presence of the bulk $\alpha$-phonon absorption superimposed to the Drude term (see Supplementary Note 1, Supplementary Table 1 and Supplementary Fig. 1 for details). Spectrally resolved transmittances are quantitatively similar to the ones measured on films belonging to the same batch in refs 46-49. By increasing the $\mathrm{THz}$ field, transmittances become more flat and, at $1 \mathrm{MV} \mathrm{cm}^{-1}$, enhance their value to $\sim 80 \%(75 \%)$ for the 60 (120)-QL films.

The integrated transmittance as a function of the $\mathrm{THz}$ electric field amplitude $E_{0}$ can be described by a phenomenological saturable absorption model:

$$
T\left(E_{0}\right)=T_{\mathrm{ns}} \frac{\ln \left[1+T_{\text {lin }} / T_{\mathrm{ns}}\left(e^{E_{0}^{2} / E_{\text {sat }}^{2}}-1\right)\right]}{E_{0}^{2} / E_{\text {sat }}^{2}}
$$

where $T_{\text {lin }}$ and $T_{\text {ns }}$ are the linear and the non-saturable integrated transmittances, and $E_{\text {sat }}$ is the $\mathrm{THz}$ electric field saturation value. If one fixes $T_{\text {lin }}$ and $T_{\mathrm{ns}}$ from the data $\left(T_{\text {lin }}=0.59 \%\right.$ and $T_{\mathrm{ns}}=0.69 \%$ for $60-\mathrm{QL}$ film, $T_{\text {lin }}=0.51 \%$ and $T_{\mathrm{ns}}=0.65 \%$ for 120-QL film), one obtains from the fit (blue point-dashed curves in Fig. 2a,b), comparable values of $E_{\text {sat }}$ for both the samples: $0.32 \mathrm{MV} \mathrm{cm}^{-1}$ for $120 \mathrm{QL}$ and $0.31 \mathrm{MV} \mathrm{cm}^{-1}$ for $60 \mathrm{QL}$, which correspond to a saturation fluence $F$ of $\sim 50 \mu \mathrm{J} \mathrm{cm}^{-2}$.

The constant value of $E_{\text {sat }}$ as measured on different thickness (60 and $120 \mathrm{QL}$ ) $\mathrm{Bi}_{2} \mathrm{Se}_{3}$ films is a clear signature that the nonlinear absorption in topological insulators is a surface property and does not depend on their bulk characteristics. Moreover, such a saturation fluence is comparable to that measured in doped graphene $\left(F=20 \mu \mathrm{J} \mathrm{cm}^{-2}\right)^{32}$.

To properly assign the nonlinear electromagnetic response observed in $\mathrm{Bi}_{2} \mathrm{Se}_{3}$ to Dirac electrons, we have investigated also the optical response of a $\left(\mathrm{Bi}_{1-x} \mathrm{In}_{x}\right)_{2} \mathrm{Se}_{3}$ film with $x=0.1$. While indium substitution does not change the crystal structure, it induces a quantum phase transition from a topological to a trivial phase for an In content larger than 0.045 (ref. 47). Moreover, the $\left(\mathrm{Bi}_{0.9} \mathrm{In}_{0.1}\right)_{2} \mathrm{Se}_{3}$ film shows, due to the band-bending effects, a gas of massive electrons having a surface density $\left(n_{\mathrm{M}}=2.5 \times 10^{13} \mathrm{~cm}^{-2}\right)$, comparable to the Dirac surface density in $\mathrm{Bi}_{2} \mathrm{Se}_{3}$ (ref. 51). At variance with $\mathrm{Bi}_{2} \mathrm{Se}_{3}$, the integrated transmittance of $\left(\mathrm{Bi}_{0.9} \mathrm{In}_{0.1}\right)_{2} \mathrm{Se}_{3}$, which is shown in Fig. 2c, is completely flat over seven decades of the $\mathrm{THz}$ electric field amplitude. Moreover, the spectrally resolved transmittances 


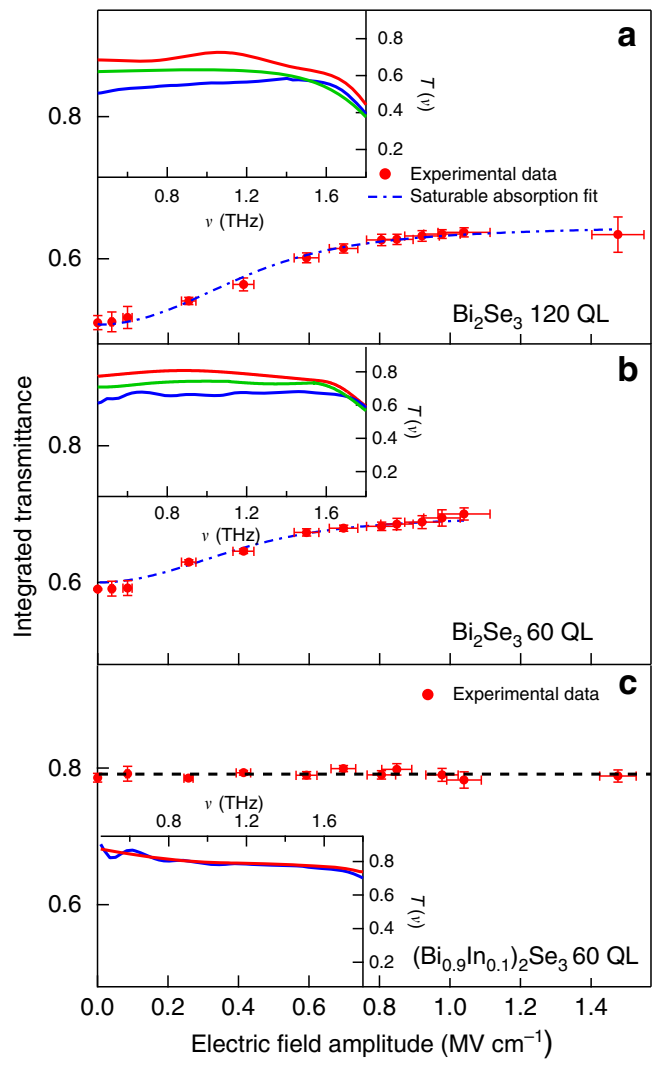

Figure 2 | THz nonlinear behaviours of the $\mathrm{Bi}_{2} \mathrm{Se}_{3}$ topological insulator. Integrated transmittance of $\mathrm{Bi}_{2} \mathrm{Se}_{3} 120-\mathrm{QL}(\mathbf{a}), \mathrm{Bi}_{2} \mathrm{Se}_{3} 60-\mathrm{QL}(\mathbf{b})$ and

$\left(\mathrm{Bi}_{0.9} \mathrm{In}_{0.1}\right)_{2} \mathrm{Se}_{3} 60-\mathrm{QL}$ (c) films, respectively, as a function of the incident $\mathrm{THz}$ electric field amplitude. Experimental data are represented by red dots. The error bars on the order of $0.5 \%$ on both the integrated transmittance and electric field amplitude correspond to the statistical fluctuations of the measured signals averaged over 100 shots of the SPARC THz source. The dashed dotted blue line corresponds to a fit with a saturable absorption model that is described in the main text. Insets: spectrally resolved transmittance curves measured (solid lines) at $1 \mathrm{MV} \mathrm{cm}^{-1}$ (red curve), $0.4 \mathrm{MV} \mathrm{cm}^{-1}$ (green curve) and $0.1 \mathrm{~V} \mathrm{~cm}^{-1}$ (blue curve) for $\mathrm{Bi}_{2} \mathrm{Se}_{3} 120 \mathrm{QL}$ (a) and $\mathrm{Bi}_{2} \mathrm{Se}_{3} 60 \mathrm{QL}$ (b). The spectrally resolved transmittance of $\left(\mathrm{Bi}_{0.9} \mathrm{In}_{0.1}\right)_{2} \mathrm{Se}_{3} 60 \mathrm{QL}$ at $0.1 \mathrm{~V} \mathrm{~cm}^{-1}$ (blue curve) and $1 \mathrm{MV} \mathrm{cm}^{-1}$ (red curve), which are superimposed in the limit of our sensitivity, is shown in the inset of $\mathbf{c}$. The slow modulation in the spectrally resolved transmittances are related to a non-perfect compensation of water absorption in the $\mathrm{THz}$ range.

both at $0.1 \mathrm{~V} \mathrm{~cm}^{-1}$ and $1 \mathrm{MV} \mathrm{cm}^{-1}$ are superimposed within our experimental sensitivity. These results undoubtedly indicate that the strong nonlinear electromagnetic response previously observed in $\mathrm{Bi}_{2} \mathrm{Se}_{3}$ must be attributed to the $2 \mathrm{D}$ gas of Dirac electrons present at the surface of topological insulators.

\section{Discussion}

In graphene, the electromagnetic-induced transparency has been interpreted in terms of a combination of the two mechanisms: harmonic generation and a strong decrease of carrier mobility (that is, an increase of carrier scattering rate), due to the opening of new scattering channels for the accelerated carriers ${ }^{28,32,33}$. To clarify the transparency microscopic mechanism in $\mathrm{Bi}_{2} \mathrm{Se}_{3}$ topological insulator, we have performed a further experiment on a 60-QL-thick film with the aim of looking for a specific harmonic signal. In particular, a pulse centred at $1 \mathrm{THz}$ with a maximum field of $\sim 300 \mathrm{kV} \mathrm{cm}^{-1}$ (selected from the broad a

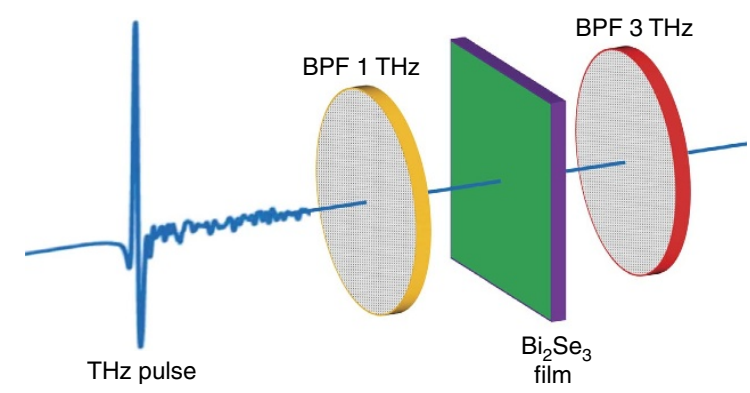

b

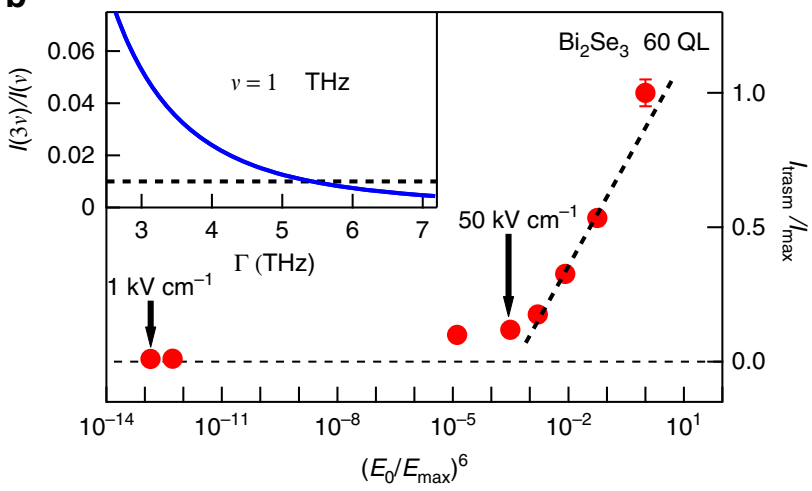

Figure 3 | Third harmonic generation in $\mathrm{Bi}_{2} \mathrm{Se}_{3}$ topological insulator. Optical scheme for a third harmonic measurement. A band-pass optical filter (BPF) selects from the broad SPARC THz spectrum a pulse centred at $1 \mathrm{THz}$ and having a full width at half maximum of $0.18 \mathrm{THz}$. This pulse, with a maximum electric field of $E_{\max }=300 \mathrm{kV} \mathrm{cm}^{-1}$, illuminates a 60 -nm-thick $\mathrm{Bi}_{2} \mathrm{Se}_{3}$ film. The transmitted intensity is collected through a filter centred at $3 \mathrm{THz}$ with a full width at half maximum of $0.36 \mathrm{THz}$, and finally measured through a pyroelectric detector (a). Above nearly $50 \mathrm{kV} \mathrm{cm}^{-1}$ the transmitted intensity (normalized to its maximum value) follows a $\left(E_{0} / E_{\max }\right)^{6}$ dependence, suggesting a third harmonic conversion process (b). In the inset of $\mathbf{b}$, the efficiency $\epsilon=I(3 v) / I(v)$ of third harmonic generation (where $\mathrm{I}(3 v)$ and $\mathrm{I}(v)$ are the transmitted intensities of a sample at a frequency $3 v$ and $v$, respectively) is represented versus the charge-carrier scattering rate $\Gamma$. The measured efficiency of $1 \%$ is obtained for $\Gamma \sim 5.5 \mathrm{THz}$.

SPARC spectrum by a band-pass $\mathrm{THz}$ filter, see the Methods section and Supplementary Information) has been used to stimulate the $60-\mathrm{QL} \mathrm{Bi}_{2} \mathrm{Se}_{3}$ film. Let us observe that at $1 \mathrm{THz}$ the SPARC source has its maximum intensity (Supplementary Fig. 2). The transmitted intensity (for the optical scheme see Fig. 3a) was collected through a band-pass filter centred at $3 \mathrm{THz}$ and finally measured through a pyroelectric detector. Both the filters (whose $\mathrm{THz}$ response is shown in Supplementary Fig. 3) have a maximum transmittance of $\sim 80 \%$, and a full width half maximum bandwidth, of $0.18 \mathrm{THz}$ (at $1 \mathrm{THz}$ ) and $0.36 \mathrm{THz}$ (at $3 \mathrm{THz}$ ). Let us note that the full width half maximum bandwidth of the $3-\mathrm{THz}$ filter is sufficiently broad to capture the third harmonic signal $(1 \rightarrow 3 \mathrm{THz})$ in $\mathrm{Bi}_{2} \mathrm{Se}_{3}$ films. By finally varying the incident intensity (that is, the incident electric field) through a couple of $\mathrm{THz}$ polarizers, we were able to measure the transmitted intensity from an incident field $E_{0}$ of $\sim 1 \mathrm{kV} \mathrm{cm}^{-1}$ to a maximum field of $300 \mathrm{kV} \mathrm{cm}^{-1}$. This intensity (normalized to its maximum value in Fig. 3b), first increases smoothly at the low-field values. Around $50 \mathrm{kV} \mathrm{cm}^{-1}$ it rapidly grows, scaling with $\left(E_{0} / E_{\max }\right)^{6}$ (black dashed line in Fig. $3 \mathrm{~b}$ ), where $E_{\max }=300 \mathrm{kV} \mathrm{cm}^{-1}$. Although, from the present measurements, we cannot exclude incoherent contribution to the $3-\mathrm{THz}$ 
signal coming from supercontinuum generation and hot-carrier emission $^{27}$, its dependence on the sixth power of $E_{0}$ is highly suggestive (see for instance ref. 55), of a third harmonic generation (THG) process. In this regard, one can note that $50 \mathrm{kV} \mathrm{cm}^{-1}$ corresponds to the field where the electromagnetic momentum gained by electrons is comparable with the Fermi momentum, that is, the threshold at which one expect strong nonlinear effects.

The efficiency of THG process can be measured by the ratio $\epsilon=I(3 v) / I(v)$, where $I(3 v) /(I(v)$ is the transmitted intensity of a sample at the frequency $3 v(v)$.This ratio at $v=1 \mathrm{THz}$ is $\sim 1 \%$ in quite good agreement with graphene $e^{28}$. $\epsilon$ depends on four parameters of the Dirac electron gas: the Fermi velocity $V_{\mathrm{F}}$, the Fermi energy $E_{\mathrm{F}}$, the Dirac charge density $n_{\mathrm{D}}$ and the actual scattering rate of charge-carrier $\Gamma$ (ref. 26). $\epsilon$ has been calculated theoretically for graphene in ref. 26 and calculation can be extended to $\mathrm{Bi}_{2} \mathrm{Se}_{3}$. Indeed, the first three parameters are well known from transport and photoemission experiments performed on films of the same batch ${ }^{47,52}: V_{\mathrm{F}}=5 \pm 1 \times 10^{7} \mathrm{~cm} \mathrm{~s}^{-1}$, $E_{\mathrm{F}}=380 \pm 10 \mathrm{meV}$ and $n_{\mathrm{D}}=3 \pm 1 \times 10^{13} \mathrm{~cm}^{-2}$. Therefore, using equation 11 in ref. 26 , a strong reduction of the efficiency from the ideal value of $1 / 3$ to $\sim 6 \%$ is already obtained at $300 \mathrm{kV} \mathrm{cm}^{-1}$ and $1 \mathrm{THz}$, if the scattering rate is set to its linear value of $3.1 \mathrm{THz}$. An efficiency of $\sim 1 \%$ is finally achieved by considering a further field renormalization of the scattering rate. Indeed, $\epsilon$ is a rapid decreasing function of $\Gamma$ (inset in Fig. 3b), and takes a value $\sim 1 \%$ when $\Gamma$ is $\sim 5.5 \mathrm{THz}$. This suggests a quite strong enhancement of the scattering rate of Dirac electrons versus $E_{0}$ as already observed for graphene in refs $25,26,32$.

In graphene, several mechanisms contribute to the scattering of Dirac electrons: electron-electron scattering, short- and longrange impurity scattering, and optical-phonon interaction ${ }^{32,56}$. The relative importance of these mechanisms depends on the Fermi energy and temperature. As a matter of fact, to a strong $\mathrm{THz}$ pulse corresponds an overall increase of the scattering rate and then a reduction of harmonic conversion efficiency. Although poor information is present about the relative importance of the different scattering mechanisms in $\mathrm{Bi}_{2} \mathrm{Se}_{3}$, one can expect that a similar enhancement of the scattering rate takes place and this renormalization may continue even at higher electric field. Let us observe that taking into account the quite large decreasing of $\Gamma$ with $T^{48}$, one can expect an enhancement of the conversion efficiency at low temperature. Moreover, $\epsilon$ could be further improved by reducing the impurity and defect scattering contributions through a better control of film-growing process.

The electromagnetic response of $\mathrm{Bi}_{2} \mathrm{Se}_{3}$ thin films has been investigated over seven decades of $\mathrm{THz}$ electric field: from $0.1 \mathrm{~V} \mathrm{~cm}^{-1}$ by means of conventional Fourier transform $\mathrm{THz}$ spectroscopy to $1.5 \mathrm{MV} \mathrm{cm}^{-1}$ using the linac-based SPARC_LAB $\mathrm{THz}$ source. We observed a strong reduction of the absorption of $\mathrm{Bi}_{2} \mathrm{Se}_{3}$ topological insulator for an increasing $\mathrm{THz}$ field that determines an electromagnetic-induced transparency in this material.

The induced transparency is determined only by the surface states of $\mathrm{Bi}_{2} \mathrm{Se}_{3}$ as films with different thickness shows exactly the same $\mathrm{THz}$ behaviour. Moreover, a similar experiment performed on a $\left(\mathrm{Bi}_{0.9} \mathrm{In}_{0.1}\right)_{2} \mathrm{Se}_{3}$ film, which presents a trivial topological phase characterized by a gas of massive electrons, shows an absorption that does not depend on the THz field amplitude. This demonstrates that the strong nonlinear effects in $\mathrm{Bi}_{2} \mathrm{Se}_{3}$ are driven by massless Dirac electrons at the surface.

Incoherent emission processes at high frequency may contribute to the induced transparency. However, as a consequence of a nonlinear stimulation at $1 \mathrm{THz}$, the transmitted signal at $3 \mathrm{THz}$ clearly scales with the sixth power of the electric field amplitude. This result suggests a THG process. The efficiency of harmonic generation is on the order of $1 \%$ at variance with the nominal value of $1 / 3$. This strong renormalization is mainly determined by the charge-carrier scattering rate. Therefore, the electromagnetic transparency in $\mathrm{Bi}_{2} \mathrm{Se}_{3}$ is generated by two concomitant effects: an intrinsic harmonic generation process and an extrinsic mobility reduction. Then, one could increase the harmonic conversion efficiency by working at low temperature and further improving the film quality.

In conclusion, the possibility to control light by light in the $\mathrm{THz}$ regime is an actual subject of intense study to implement compelling applications in $\mathrm{THz}$ technology, such as ultrafast $\mathrm{THz}$ tabletop sources, quantum cascade lasers and ultrafast $\mathrm{THz}$ communications based on optical bistability. In this regard, the strong nonlinear $\mathrm{THz}$ properties observed in $\mathrm{Bi}_{2} \mathrm{Se}_{3}$ Dirac material could open promising perspectives in the tumultuous field of $\mathrm{THz}$ technologies.

\section{Methods}

Topological insulator films. The high-quality $\left(\mathrm{Bi}_{1-x} \mathrm{In}_{x}\right)_{2} \mathrm{Se}_{3}$ thin films were prepared by molecular beam epitaxy using the standard two-step growth method developed at the Department of Physics and Astronomy Rutgers, The State University of New Jersey ${ }^{52,53}$. The $10 \times 10-\mathrm{mm}^{2} \mathrm{Al}_{2} \mathrm{O}_{3}$ substrates were first cleaned by heating to $750{ }^{\circ} \mathrm{C}$ in an oxygen environment to remove organic surface contamination. An initial 3 QLs of $\mathrm{Bi}_{2} \mathrm{Se}_{3}$ were deposited on the substrates at $110^{\circ} \mathrm{C}$, which was then followed by heating to $220^{\circ} \mathrm{C}$ helping further to achieve the target thickness. The crystallization of the films during the growth was monitored by reflection high-energy electron diffraction. The Se:(Bi/In) optimal flux ratio was 10:1 for the deposition. A pre-control Bi/In flux ratio was performed to achieve the desired In concentration. Once the films were cooled, they were removed from the vacuum chamber, and vacuum-sealed in plastic bags within $2 \mathrm{~min}$, then shipped to the University of Rome.

High-field THz generation and measurements. The ultra-relativistic electron bunches $650 \mathrm{pC}$ charged with a time duration of $\simeq 120 \mathrm{fs}$ and $10 \mathrm{~Hz}$ of repetition rate were used to generate nearly single-cycle $\mathrm{THz}$ pulses as coherent transition radiation at SPARC_LAB ${ }^{12,14}$. The THz electric field in the focal point was estimated in two ways. The first estimate consists in using the nominal sensitivity of the pyroelectric detector $\left(140 \mathrm{kV} \mathrm{W}^{-1}\right)$ that has been experimentally tested at $970 \mathrm{GHz}$ through a Schottky Diode (Virginia Diode) emitting a power of $1 \mathrm{~mW}$. In the second estimate, we calculated the $\mathrm{THz}$ electric field in the focal point produced by the CTR SPARC source by a THz transport simulation program ${ }^{57}$. In this calculation, we take into account the actual (finite) size of CTR target, the actual transmittance of the $z$-cut quartz window and the optical properties of the propagation optics. Both the methods provide comparable $\mathrm{THz}$ electric fields in the focal point having a highest $\mathrm{THz}$ field amplitude of $1.5 \mathrm{MV} \mathrm{cm}^{-1}$.

The $\mathrm{THz}$ radiation coming from the CTR source was separated in two beams by a high-resistivity $\mathrm{Si}$ beam splitter at $45^{\circ}$ (Fig. 1). The transmitted beam propagates towards the films, while that reflected one towards a pyroelectric detector. The THz-integrated spectra were measured placing just behind the films a pyroelectric detector, meanwhile the $\mathrm{THz}$ spectra have been measured by a step-scan Michelson interferometer, having a $24-\mu \mathrm{m}$ Mylar pellicle beam splitter. All radiation channels were equipped with THz-I-BNC GENTEC-EO pyroelectric detectors.

We used a differential detection technique to reduce the shot-to-shot fluctuations of the SPARC THz source. In particular, both the integrated signal and the spectrally resolved one were normalized to the signal as measured by the reference pyroelectric detector. This technique provides an error bars on the transmittance (both integrated and spectrally resolved) on the order of $0.5 \%$.

The $\mathrm{THz}$ filters used in the harmonic detection experiment have been acquired from Tydex (http://www.tydexoptics.com). Their optical response controlled by a Michelson interferometer are reported in Supplementary Fig. 3. The THz radiation transmitted by the series 1-THz filter, 60-QL film and 3-THz filter has been finally collected using a THz-I-BNC pyroelectric by GENTEC-EO.

\section{References}

1. Boyd, R. W. Nonlinear Optics 3rd edn (Academic, 2008).

2. Fausti, D. et al. Light-induced superconductivity in a stripe-ordered cuprate. Science 331, 189-191 (2011).

3. Rini, M. et al. Control of the electronic phase of a manganite by mode-selective vibrational excitation. Nature 449, 72-74 (2007).

4. Rulliere, C. (ed.) Femtosecond Laser Pulses (Springer, 2005).

5. Chen, Y.-C. et al. Ultrafast optical switching properties of single-wall carbon nanotube polymer composites at 1.55 m. App. Phys. Lett. 81, 975-977 (2002)

6. US Department of Energy (ed.). Opportunities in THz Science. Report of a DOE-NSF-NIH Workshop, 12-14 February 2004 (Arlington, VA, USA, 2004), OSTI Identifier: 899222. 
7. Hebling, J., Almási, G., Kozma, I. Z. \& Kuhl, J. Velocity matching by pulse front tilting for large area THz-pulse generation. Opt. Express 10, 1161-1166 (2002).

8. Matsuura, S., Tani, M. \& Sakai, K. Generation of coherent terahertz radiation by photomixing in dipole photoconductive antennas. App. Phys. Lett. 70, 559-561 (1997).

9. Vicario, C. et al. High efficiency THz generation in DSTMS, DAST and OH1 pumped by Cr:forsterite laser. Opt. Express 23, 4573-4580 (2015).

10. Carr, G. L. et al. High-power terahertz radiation from relativistic electrons Nature 420, 153-156 (2002).

11. Chiadroni, E. et al. Characterization of the $\mathrm{THz}$ radiation source at the Frascati linear accelerator. Rev. Sci. Instrum. 84, 022703 (2013).

12. Ferrario, M. et al. SPARC-LAB present and future. Nucl. Instrum. Methods Phys. Res. Sect. B 309, 183-188 (2013).

13. Ferrario, M. et al. Experimental demonstration of emittance compensation with velocity bunching. Phys. Rev. Lett. 104, 054801 (2010).

14. Chiadroni, E. et al. The SPARC linear accelerator based terahertz source. Appl. Phys. Lett 102, 09410 (2013).

15. Perucchi, A. et al. The TeraFERMI terahertz source at the seeded FERMI free-electron-laser facility. Rev. Sci. Instrum. 84, 022702 (2013).

16. Padilla, W. J. et al. Electrically resonant terahertz metamaterials: theoretical and experimental investigations. Phys. Rev. B 75, 041102 (2007).

17. Limaj, O. et al. Superconductivity-induced transparency in terahertz metamaterials. ACS Photon. 1, 570-575 (2014).

18. D'Apuzzo, F. et al. Resonating terahertz response of periodic arrays of subwavelength apertures. Plasmonics 10, 45-50 (2015).

19. Razzari, L. et al. Nonlinear ultrafast modulation of the optical absorption of intense few-cycle terahertz pulses in n-doped semiconductors. Phys. Rev. B 79, 19320 (2009).

20. Hoffmann, M. C. \& Turchinovich, D. Semiconductor saturable absorbers for ultrafast terahertz signals. App. Phys. Lett. 96, 151110 (2010).

21. Hebling, J. et al. Observation of nonequilibrium carrier distribution in $\mathrm{Ge}, \mathrm{Si}$, and GaAs by terahertz pumpterahertz probe measurements. Phys. Rev. B 81, 035201 (2010)

22. Dong, H., Conti, C. \& Biancalana, F. Terahertz relativistic spatial solitons in doped graphene metamaterials. J. Phys. B At. Mol. Opt. Phys. 46, 155401 (2013).

23. Mikhalov, S. A. \& Ziegler, K. Nonlinear electromagnetic response of graphene: frequency multiplication and the self-consistent-field effects. J. of Phys. Condens. Matter 20, 384204 (2008).

24. Mikhailov, S. A. Non-linear electromagnetic response of graphene. Europhys Lett. 79, 27002 (2007)

25. Al-Naib, I., Poschmann, M. \& Dignam, M. M. Optimizing third-harmonic generation at terahertz frequencies in graphene. Phys. Rev. B 91, 205407 (2015).

26. Mikhailov, S. A. Quantum theory of third-harmonic generation in graphene. Phys. Rev. B 90, 241301 (2014).

27. Cheng, J. L., Vermeulen, N. \& Sipe, J. E. Third order nonlinearity of graphene: effects of phenomenological relaxation and finite temperature. Phys. Rev. B 91, 235320 (2015)

28. Bowlan, P., Martinez-Moreno, E., Reimann, K., Elsaesser, T. \& Woerner, M. Ultrafast terahertz response of multilayer graphene in the nonperturbative regime. Phys. Rev. B 89, 041408 (2014).

29. Novoselov, K. S. A. et al. Two-dimensional gas of massless Dirac fermions in graphene. Nature 438, 197-200 (2005).

30. Geim, A. K. \& Novoselov, K. S. The rise of graphene. Nat. Mater. 6, 183-191 (2007).

31. Hendry, E., Hale, P. J., Moger, J., Savchenko, A. K. \& Mikhailov, S. A. Coherent nonlinear optical response of graphene. Phys. Rev. Lett. 105, 097401 (2010).

32. Hwang, H. Y. et al. Nonlinear THz conductivity dynamics in p-type CVD-grown graphene. J. Phys. Chem. B 117, 15819-15824 (2013).

33. Paul, M. J. et al. High-field terahertz response of graphene. New J. Phys. 15, 085019 (2013).

34. Dragoman, M. et al. Millimeter-wave generation via frequency multiplication in graphene. App. Phys. Lett. 97, 093101 (2010).

35. Liang, T. et al. Ultrahigh mobility and giant magnetoresistance in the Dirac semimetal $\mathrm{Cd}_{3} \mathrm{As}_{2}$. Nat. Mater. 14, 280-284 (2015).

36. Liu, Z. K. et al. Discovery of a three-dimensional topological dirac semimetal, $\mathrm{Na}_{3} \mathrm{Bi}$. Science 343, 864-867 (2014).

37. Moore, J. E. The birth of topological insulators. Nature 464, 194-198 (2010).

38. Zhang, $\mathrm{H}$. et al. Topological insulators in $\mathrm{Bi}_{2} \mathrm{Se}_{3}, \mathrm{Bi}_{2} \mathrm{Te}_{3}$ and $\mathrm{Sb}_{2} \mathrm{Te}_{3}$ with a single Dirac cone on the surface. Nat. Phys. 5, 438-442 (2009).

39. Di Pietro, P. et al. Optical conductivity of bismuth-based topological insulators. Phys. Rev. B 86, 045439 (2012).

40. Hasan, M. Z. \& Kane, C. L. Colloquium: topological insulators. Rev. Mod. Phys. 82, 30453067, 2010).

41. Kitaev, A. \& Laumann, C. Topological phases and quantum computation. Preprint at http://arxiv.org/abs/0904.2771 (2009).
42. Zhang, X., Wang, J. \& Zhang, S. C. Topological insulators for high-performance terahertz to infrared applications. Phys. Rev. B 82, 245107 (2010).

43. Pesin, D. \& MacDonald, A. H. Spintronics and pseudospintronics in graphene and topological insulators. Nat. Mater. 11, 409-416 (2012).

44. Shuvaev, A. M. et al. Giant magneto-optical Faraday effect in HgTe thin films in the terahertz spectral range. Phys. Rev. Lett. 106, 107404 (2011).

45. Hancock, J. N. et al. Surface state charge dynamics of a high-mobility three-dimensional topological insulator. Phys. Rev. Lett. 107, 136803 (2011).

46. Aguilar, R. V. et al. Terahertz response and colossal Kerr rotation from the surface states of the topological insulator $\mathrm{Bi}_{2} \mathrm{Se}_{3}$. Phys. Rev. Lett. 108, 087403 (2012).

47. Wu, L. et al. A sudden collapse in the transport lifetime across the topological phase transition in $\left(\mathrm{Bi}_{1-x} \mathrm{In}_{x}\right)_{2} \mathrm{Se}_{3}$. Nat. Phys. 9, 410-414 (2013).

48. Autore, M. et al. Plasmon-phonon interactions in topological insulator microrings. Adv. Opt. Mater. 3, 1257-1263 (2015).

49. Di Pietro, P. et al. Observation of Dirac plasmons in a topological insulator. Nat. Nanotechnol. 8, 556-560 (2013).

50. Autore, M. et al. Observation of magnetoplasmons in $\mathrm{Bi}_{2} \mathrm{Se}_{3}$ topological insulator. ACS Photon. 2, 1231-1235 (2015).

51. Brahlek, M. et al. Topological-metal to band-insulator transition in $\left(\mathrm{Bi}_{1-x} \mathrm{In}_{x}\right)_{2} \mathrm{Se}_{3}$ thin films. Phys. Rev. Lett. 109, 186403 (2012).

52. Bansal, N. et al. Thickness-independent transport channels in topological insulator $\mathrm{Bi}_{2} \mathrm{Se}_{3}$ thin films. Phys. Rev. Lett. 109, 116804 (2012).

53. Bansal, N. et al. Epitaxial growth of topological insulator $\mathrm{Bi}_{2} \mathrm{Se}_{3}$ film on Si (111) with atomically sharp interface. Thin Solid Films 520, 224-229 (2011).

54. Stauber, T., Gomez-Santos, G. \& Brey, T. Spin-charge separation of plasmonic excitations in thin topological insulators. Phys. Rev. B 88, 205427 (2013).

55. Matsunaga, M. et al. Light-induced collective pseudospin precession resonating with Higgs mode in a superconductor. Science 345, 1145-1149 (2014).

56. Winnerl, S. et al. Time-resolved spectroscopy on epitaxial graphene in the infrared spectral range: relaxation dynamics and saturation behaviour. J. Phys. Condens. Matter 25, 054202 (2013).

57. Casalbuoni, S., Schmidt, B., Schmuser, P., Arsov, V. \& Wesc, S. Ultrabroadband terahertz source and beamline based on coherent transition radiation. Phys. Rev. Spec. Top. Accel. Beams 12, 030705 (2009).

\section{Acknowledgements}

We thank S.A. Mikhailov for useful discussion about harmonic generation theory. M.B., N.K. and S.O. thank for the financial support the Office of Naval Research (N000141210456) and the Gordon and Betty Moore Foundations EPiQS Initiative through grant GBMF4418. A.P. acknowledges support by Italian Ministry of Research (MIUR) program FIRB Futuro in Ricerca grant no. RBFR10PSK4.

\section{Author contributions}

M.B., N.K. and S.O. fabricated and characterized the $\left(\mathrm{Bi}_{1-x} \mathrm{In}_{x}\right)_{2} \mathrm{Se}_{3}$ films. F.G., E.C., A.R., M.C.-G. and S.L. carried out the THz experiments and data analysis. M.B., E.C., M.C., D.D.G., G.D.P., M.F., R.P., C.V., F.V., A.C., A.M. and M.P. managed the SPARC_LAB photoinjector during the THz experiments. S.L. planned and managed the project with inputs from all the co-authors. F.G., A.P. and S.L. wrote the manuscript. All authors extensively discussed the results.

\section{Additional information}

Supplementary Information accompanies this paper at http://www.nature.com/ naturecommunications

Competing financial interests: The authors declare no competing financial interests

Reprints and permission information is available online at http://npg.nature.com/ reprintsandpermissions/

How to cite this article: Giorgianni, F. et al. Strong nonlinear terahertz response induced by Dirac surface states in $\mathrm{Bi}_{2} \mathrm{Se}_{3}$ topological insulator. Nat. Commun. 7:11421 doi: 10.1038/ncomms11421 (2016).

This work is licensed under a Creative Commons Attribution 4.0 International License. The images or other third party material in this article are included in the article's Creative Commons license, unless indicated otherwise in the credit line; if the material is not included under the Creative Commons license, users will need to obtain permission from the license holder to reproduce the material. To view a copy of this license, visit http://creativecommons.org/licenses/by/4.0/ 